\title{
Can we predict agitation in patients with suicide attempts in the emergency department?
}

\author{
Ayhan Akoz ${ }^{1}$, Sultan Tuna Akgol Gur², Elif Oral ${ }^{3}$, Ummu Zeynep Avsar ${ }^{4}$, Mucahit Emet ${ }^{2}$
}

1. Adnan Menderes University Medicine Faculty Department of Emergency Medicine, Aydin, Turkey

2. Ataturk University Medicine Faculty Department of Emergency Medicine, Erzurum, Turkey

3. Ataturk University Medicine Faculty Department of Psychiatry, Erzurum, Turkey

4. Ataturk University Medicine Faculty Department of Medical Education, Erzurum, Turkey

\begin{abstract}
Background: The agitation in patients presenting to the emergency department (ED) after suicide attempts is common and an important problem.
\end{abstract}

Objective: To establish whether we can predict agitated patients among suicide attempt patients in ED.

Methods: This is a cross-sectional observational study of adult suicide attempt events in ED. Information was collected prospectively on a specially designed data-collection form. Patients aged 16 years old and above who presented to the ED for care due to suicide attempts were included in the study. Suicide attempts were grouped as aggressive and non-aggressive attempts.

Results: A total of 533 patients were included. Forty-three of these patients had agitation in ED (8\%). Non-aggressive suicide attempts were referred to psychiatry services more than aggressive ones $(73.6 \%, \mathrm{n}=345$ vs $32.8 \%, \mathrm{n}=21, \mathrm{P}<0.0001)$. Agitation in ED and being male increased aggressive suicide attempt risk 3.5 (95\% CI:1.6-7.6) and 3.2 times (95\% CI:1.8-5.5), respectively. Agitation was statistically more frequent among these patients: those on antidepressant overdose, with previous suicide attempt; with aggressive suicide attempt; and those with confusion; and unconsciousness $(\mathrm{P}<0.05)$.

Conclusion: Patients who attempted suicide and whose risk of harm to others included those with: antidepressant overdose, aggressive suicide attempt and the unconscious. Response teams should be prepared for these subgroups.

Keywords: Agitation, suicide attempt, deliberate self-harm.

DOI: http://dx.doi.org/10.4314/ahs.v16i3.25

Cite as: Akoz, A, Gur STA, Oral E, Avsar UZ, Emet M. Can we predict agitation in patients with suicide attempts in the emergency department? Afri Health Sci 2016;16(3): 831-837. DOI: http:/ / dx.doi.org/10.4314/abs.v16i3.25

\section{Introduction}

Suicide attempt is a common health issue which is poorly understood and arouses ambivalent feelings in health professionals ${ }^{1}$. Emergency departments (EDs) can serve as an important link to mental health care and to the ED doctors who are at the frontline in the assessment of suicide attempts ${ }^{2,3}$.

The primary problem that requires a response from response teams for behavioural emergencies in EDs is de-

\section{Corresponding author:}

Ayhan Akoz,

Department of Emergency Medicine,

Adnan Menderes University Medical Faculty,

09020, Aydin, Turkey

Tel: +902562181800

Fax:+902562146495

E-mail: akozayhan@gmail.com liberate self-poisoning or self-harm, with a rate of $38 \% 0^{4}$. Furthermore, agitated patients are uncooperative or unable to give a relevant history, forcing clinicians to make decisions based on limited information ${ }^{5}$. Therefore, it is important to determine which of these patients will be agitated in EDs, as this will affect resistance to treatment, as well as safety of medical personnel.

The primary purpose of this cross-sectional study was to determine whether we can foresee which patients will show agitation among those who seek care in EDs due to suicide attempts. Our second purpose was to analyse patients according to suicide type. We analysed patients according to the time series in a second article, as our data in that four-year study is extensive and detailed.

\section{Methods}

All aspects of the study protocol were authorized by the medical ethics committee and the local health authorities prior to initiation of this study (Date: 21.04.2009; No:

African Health Sciences Vol 16 Issue 3, September 2016 
B.30.2.ATA.0.01.00/132). Informed consent was obtained from each patient.

Study site: This was a cross-sectional observational study of adult suicide attempt events brought to a 1,300-bed university hospital located in Erzurum in the Eastern region of Turkey between May 2008 and January 2012. The population of the city centre of Erzurum is 383,000, and the population of the province is 769,000 . Although our hospital primarily provides health services for individuals who live in the city centre, it also provides services for individuals from the counties and villages of Erzurum and 11 neighbouring cities, comprising a total of four million individuals ${ }^{6}$. The hospital provides healthcare and emergency treatment for all illnesses and accidents. Our hospital did not have a self-harm team at the time of the study.

Inclusion and exclusion criteria: Patients aged 16 years and above and who presented to the ED for care due to suicide attempts by oral ingestion, or suicidal self-injury, were included in the study. Patients younger than 16 years of age who were brought to the paediatric ED for care due to suicide attempts by oral ingestion, drug intoxication, non-suicidal accidents and self-injuries were excluded.

Patients who engaged in mutilation without suicidal intent and repetitive superficial bodily harm without suicidal intent were excluded. Patients who were unable to fill out the study form at the time of arrival at the ED, those for whose data could not be obtained via relatives, and those who left the ED without filling out the study form were also excluded.

Study design: Information was collected prospectively on a specially designed data collection form. It included age, gender, address, stated date and time of overdose, time of admission, type of suicide attempt, time of discharge, type of the ingested drug, whether or not other drugs had been co-ingested, chief complaints, any history of psychiatric illnesses in the patient or his/her firstdegree relatives, previous suicidal attempts by the patient or his/her first-degree relatives, and previous psychiatry polyclinic admission in the last six months.

Definitions: Agitation is a group of disruptive, verbal, and/or motor behaviours that jeopardize the safety of a subject, his/her relatives, and/or the response team, thereby hindering medical care ${ }^{7}$. The Agitated Behaviour Scale (ABS) was used to define agitation by emergency physicians $^{8}$. A score of 22 or more was identified as agitated.

A suicide attempt is defined as an act with a non-fatal outcome in which an individual deliberately ingests a substance in excess of the prescribed or generally recognized therapeutic dosage 9 . Suicide attempts are divided into two groups, namely those including and those excluding violence. Violent methods (aggressive suicides) include hanging, jumping off a tall building, self-burning, driving a car off a cliff, and using sharp objects or firearms to harm oneself. Nonviolent methods (non-aggressive suicides) include attempts made through drug ingestion and the use of $\operatorname{gas}^{10}$. Table 1 presents patients' suicide attempt methods. The co-ingestion of drugs from different classes was accepted as multi-drug ingestion. The co-ingestion of alcohol or tetrahydrocannabinol was not accepted as multi-drug ingestion. In the study form used for each patient, there were 35 questions. Six of these included sociodemographic data, and five of them included psychological data. The rest included medical data of the patient.Answering the sociodemographic section of the patient lasted at most five minutes.

Data analysis: SPSS.19 (Statistical Package for Social Sciences) for Mac (SPSS Inc., Chicago, IL, USA) program was used to analyse the data. The mean values were shown together with standard deviations and expressed as means and 95\% confidence intervals (CI). Statistical analysis of the categorical variables was performed using the chi-square test or Fisher's exact test, and the analysis of the numerical variables was performed using the t-test or the Mann-Whitney $U$ test. The variables were tested for normality using the Kolmogorov-Smirnov test. The data were divided into groups, and regression analysis was performed. A p-score of $<0.05$ was accepted as statistically significant.

\section{Results}

\section{Baseline socio-demographic and clinical character- istics}

Of the 533 patients enrolled in the study, $66.8 \%(n=356)$ were female, and patients' mean age was $25.7 \pm 9.9$ years (min 14, max 88). This represented $0.24 \%$ of all ED admissions for the study period. As noted previously, the suicide types are shown in Table 1. 
Table 1. The patients according to the suicide attempt methods

\begin{tabular}{|l|l|l|}
\hline Type of suicide & $\mathbf{n ~ ( \% )}$ & $\mathbf{9 5 \%} \mathbf{C I}$ \\
\hline Aggressive attempt & $\mathbf{7 2}(\mathbf{1 3 . 5} \% \mathbf{)}$ & $\mathbf{1 0 . 9 - 1 6 . 7}$ \\
\hline Hanging & $9(1.7 \%)$ & $0.9-3.2$ \\
\hline Cutting & $23(4.3 \%)$ & $2.9-6.4$ \\
\hline Firearm & $8(1.5 \%)$ & $0.7-2.9$ \\
\hline Drug overdose + Cutting & $8(1.5 \%)$ & $0.7-2.9$ \\
\hline Gas inhalation + Cutting & $2(0.4 \%)$ & $0.1-1.4$ \\
\hline Jumping & $18(3.4 \%)$ & $2.2-5.3$ \\
\hline Drinking Corrosives & $4(0.8 \%)$ & $0.3-1.9$ \\
\hline $\begin{array}{l}\text { Non-aggressive } \\
\text { attempt }\end{array}$ & $\mathbf{4 6 1 ( 8 6 . 5 \% )}$ & $\mathbf{8 3 . 3 - 8 9 . 1}$ \\
\hline Drug overdose & $459(86.1 \%)$ & $82.9-88.8$ \\
\hline Gas inhalation* & $2(0.4 \%)$ & $0.1-1.4$ \\
\hline Total & 533 & 100 \\
\hline
\end{tabular}

*Natural gas or liquefied petroleum gas

The distribution of the patients who were brought in due to drug intoxication is presented in Table 2 according to drug type. A psychiatric consultation was requested for $366(68.3 \%)$ patients in the ED. There was a history of psychiatric clinic admission within the previous six months in $121(22.7 \%)$ patients. Of the 533 patients, 396 $(74.3 \%)$ were hospitalized, $82(15.4 \%)$ were discharged from the ED, and $55(10.3 \%)$ left the hospital. Moreover, $6.2 \%(n=4)$ of aggressive suicide attempt victims and $10.9 \%(n=51)$ of non-aggressive suicide attempt victims did not agree to the evaluation.

Table 2. The distribution of the drug intoxicated patients according to the drugs

\begin{tabular}{|l|l|c|}
\hline Drugs & $\mathbf{n}(\mathbf{\%})$ & $\mathbf{9 5 \%} \mathbf{C I}$ \\
\hline Organophosphate & $6111.4 \%$ & $9-14.4$ \\
\hline Antidepressant & $135(25.3 \%)$ & $21.8-29.2$ \\
\hline Analgesic* & $108(20.3 \%)$ & $17-23.4$ \\
\hline Antihypertensive & $26(4.9 \%)$ & $3.4-7.1$ \\
\hline Salicylate & $19(3.6 \%)$ & $2.3-5.6$ \\
\hline Paracetamol & $91(17.1 \%)$ & $14.1-20.1$ \\
\hline Antibiotic & $68(12.8 \%)$ & $10.2-15.9$ \\
\hline Multidrug & $304(57 \%)$ & $52.9-61.2$ \\
\hline Ethanol in blood & $10(9.1 \%)$ & $1-3.4$ \\
\hline THC** in urine & $11(2.1 \%)$ & $1.2-3.7$ \\
\hline Benzodiazepine in urine & $11(25.3 \%)$ & $1.2-3.7$ \\
\hline
\end{tabular}

*Analgesics other than Paracetamol; ** tetrahydrocannabinol 
Suicide attempts by antidepressant ingestion were more common among females $(30.9 \% n=110$, vs. $19.8 \% n=35$, $\mathrm{p}=0.007$, and ethanol ingestion was more common among males $(4.5 \% \mathrm{n}=8$, vs. $0.6 \% \mathrm{n}=2, \mathrm{p}=0.002)$.

Fatigue, nausea, vomiting, stomach aches, and stupor were significantly higher among the non-aggressive suicide attempt patients. Loss of consciousness and agitation were significantly higher among the aggressive patients $(p<0.05$ for each). Psychiatric consultation was requested more frequently for the non-aggressive suicide attempt patients at the $\operatorname{ED}(73.6 \% \mathrm{n}=345$, vs. $32.8 \%$ $\mathrm{n}=21, \mathrm{p}<0.0001)$.

According to the results of the analysis of loss of consciousness, agitation, and psychiatric admission within the previous six months with the enter method using logistic regression analysis, men were 3.2 times more likely to have aggressive suicide attempts (95\% CI: 1.8-5.5) and 3.5 times more likely to be agitated in the ED (95\% CI: 1.6-7.6).

In which patients is agitation more frequent among those brought to the ED following a suicide attempt? Agitation was found in 43 of 533 patients $(8.06 \%)$. The ABS scores of the agitated and non-agitated patients were $31.2 \pm 8.7$ and $15.1 \pm 6.6$, respectively $(\mathrm{p}<0.001)$. It was more frequent in females than males who were brought due to drug intoxication only ( $n=288.6 \%$, vs. $n=32.1 \%$, $\mathrm{p}=0.010)$. The differences between agitated patients and non-agitated suicide attempt patients are presented in Table 3.

\section{Table 3. The significant differences between agitated and non-agitated suicide attempt patients.}

\begin{tabular}{|l|l|l|l|}
\hline Characteristics & $\begin{array}{l}\text { Agitation (+) } \\
\text { n (\%) }\end{array}$ & $\begin{array}{l}\text { Agitation (-) } \\
\mathbf{n}(\mathbf{\%})\end{array}$ & $\mathbf{P}$ \\
\hline Antidepressant overdose & $22(15.2 \%)$ & $21(5.4 \%)$ & $\mathrm{P}<0.0001$ \\
\hline Previous suicide attempt & $11(13.6 \%)$ & $32(7.1 \%)$ & $\mathrm{P}=0.048$ \\
\hline Aggressive suicide attempt & $12(18.8 \%)$ & $31(6.6 \%)$ & $\mathrm{P}<0.0001$ \\
\hline Confusion & $22(11.6 \%)$ & $21(6.1 \%)$ & $\mathrm{P}=0.025$ \\
\hline Unconsciousness & $12(21.4 \%)$ & $31(6.5 \%)$ & $\mathrm{P}<0.0001$ \\
\hline *ABS scores & $\mathbf{3 1}$ & $\mathbf{1 5}$ & $\mathrm{P}<0.00 \mathrm{I}$ \\
\hline
\end{tabular}

*Agitated Behaviour Scale

Of these variables, when antidepressant ingestion, previous suicide attempt, suicide type, and loss of conscious- ness were analysed with the 'Enter method' using logistic regression analysis, the parameters increasing the likelihood of agitation at the ED are shown in Table 4.

Table 4: Multivariate logistic regression analysis for the risk of agitation in ED

\begin{tabular}{|l|l|l|l|l|}
\hline Characteristics & $\mathrm{P}$ & OR & $95 \% \mathrm{CI}$ & \\
\hline Antidepressant overdose & $<0.0001$ & 4.170 & 1.955 & 8.894 \\
\hline Previous suicide attempt & 0.148 & 1.765 & 0.818 & 3.812 \\
\hline $\begin{array}{l}\text { Method of suicide (aggressive vs. } \\
\text { nonaggressive) }\end{array}$ & $<0.0001$ & 5.365 & 2.270 & 12.677 \\
\hline Unconsciousness & 0.014 & 2.631 & 1.218 & 5.681 \\
\hline
\end{tabular}




\section{Discussion}

Classification for suicidal patients may be aggressive and non-aggressive suicide attempts. The causes for suicide attempts also differ in studies performed with these groups. In general, intentions behind superficial self-mutilation (such as self-cutting) differ from those in self-poisoning ${ }^{11}$. For adolescent patients, the most common reasons given were "escape" for self-poisoning and "depression" for self-cutting. In one study, half of the female cutters wanted to punish themselves, while adults with superficial self-mutilation gave reasons such as emotional distress ${ }^{12}$. We consider aggressive suicide attempts to have a higher risk $^{1}$. In our study, we determined two factors that increased the likelihood of aggressiveness among suicide attempt patients brought to the ED: being male and being agitated. Mortality, morbidity, and risk of suicide reattempts may also be higher in these patients. Another issue is the need to prevent high-risk suicide attempt patients from being overlooked at the ED. In light of our findings, we suggest that males who make non-aggressive suicide attempts, such as drug ingestion or gas poisoning, should have their self-inflicted injuries examined after all clothes have been removed if they are agitated in the ED, when a medical history cannot be obtained, or when the history is not reliable, and they must also be examined for trauma (for aggressive suicide).

In England, individuals who had not been assessed by a psychiatrist were more likely to have been "difficult," to have presented at night, to have injured rather than poisoned themselves, and to have previously harmed themselves ${ }^{1,13,14}$. In our study, a psychiatric consultation was requested more frequently for non-aggressive suicide attempt patients at the ED. We attribute this condition to the fact that either the general condition of aggressive suicide attempt patients is poorer or that they are unconscious, and psychiatric assessments are made in the clinics in which they are hospitalized. The clinics in which patients with impaired general conditions are hospitalized usually treat the patients for problems related to the clinics' areas of expertise but neglect the psychiatric assessment, and then discharge the patients. We believe the addition of a routine psychiatric assessment to the clinics' treatment algorithms would decrease repeated and more risky suicide attempts.
Agitation is a severe form of anxiety associated with motor restlessness ${ }^{15}$. Agitation has not previously been investigated in patients brought to an ED following suicide attempts. Many difficulties are encountered in the diagnosis and treatment of agitated patients in an ED. First, these patients may refuse communication with their healthcare providers and relatives, and a correct diagnosis may not be made, as an anamnesis cannot be obtained. Second, these patients may display verbal or physical violence towards healthcare providers and other patients ${ }^{16}$. Third, it is challenging to perform the related treatments, especially a nasogastric tube insertion. In our study, agitation was significantly higher in multiple suicide attempt patients, in women presenting with drug intoxication, and suicide attempt patients who used aggressive methods. We believe that the higher rate of agitation in women is related to their more frequent use of antidepressants. Agitation may be more common among males when their general condition is poorer, and unconsciousness is more frequent when they present to an ER due to their preference for aggressive suicide methods more often than women.

Agitated patients are also the primary source of injury for themselves, other patients and healthcare providers during ambulance transport ${ }^{17}$. Peroral or IV administration of sedating agents to the patients in the aforementioned risk groups may prevent potential injuries during their transport. Furthermore, it is possible to prevent the physical trauma-related complications that may develop at an ED if we can predict patients with suicide attempts who carry the risk of agitation, and we can then prepare healthcare providers and security staff. In our study, agitation was found to be more frequent in patients who had antidepressant intoxication, a history of previous suicide attempts, an aggressive suicide attempt, and loss of consciousness. Therefore, more attention must be paid when approaching these patient groups.

In our study, multivariate logistic regression analysis showed that antidepressant ingestion increased the likelihood of agitation fourfold, admission to the ED following an aggressive suicide attempt fivefold, and unconsciousness at the time of arrival to the ED threefold. We believe that increasing the number of security staff at the ED prior to examination and treatment of these patients, 
taking these patients into an isolated room, and taking a more careful approach by informing healthcare personnel before examination are necessary measures to be taken.

\section{Limitations}

Our study was based on self-reported data that was assumed to be accurate. The data was obtained from the patients and their relatives or friends. Therefore, incorrect data may have been obtained. Some cases who did not give a correct anamnesis to conceal their suicide attempts may have been overlooked. Patients' psychiatric diagnoses and mood disorders were not analysed in detail, as this study investigated suicide patients from the perspective of ED physicians. Agitation was evaluated by emergency physicians; thus if the Agitated Behaviour Scale was completed by psychiatrists, it might have influenced our results. In our patients, agitation may be associated with delirium as a result of overdose or drug toxicity, including alcohol, rather than an acute psychiatric pathology. We did not examine the aetiology of agitation in our patients.

\section{Conclusion}

Categorizing suicide attempt patients who are brought to an ED as aggressive and non-aggressive attempt patients, may be a logical option. Suicide attempt patients likely to harm themselves and others include those with: antidepressant overdose, aggressive suicide attempt and the unconscious. Response teams should be prepared for these subgroups.

\section{Conflict of interest}

The authors declare no conflict of interest.

\section{References}

1. Skegg K. Self-harm. Lancet. 2005; 366(9495): 1471-83.

2. Ting SA, Sullivan AF, Miller I, Espinola JA, Allen MH, Camargo CA Jr, et al. Multicenter study of predictors of suicide screening in emergency departments. Academic Emergency Medicine. 2012; 19(2): 239-43.

3. Rhodes AE, Bethell J, Spence J, Links PS, Streiner DL, Jaakkimainen RL. Age-sex differences in medicinal selfpoisonings: a population-based study of deliberate intent and medical severity. Social Psychiatry And Psychiatric Epidemiology. 2008; 43(8): 642-52.

4. Downes MA, Healy P, Page CB, Bryant JL, Isbister
GK. Structured team approach to the agitated patient in the emergency department. Emergency Medicine Australasia. 2009; 21(3): 196-202.

5. Currier GW, Litts D, Walsh P, Schneider S, Richardson T, Grant W, et al. Evaluation of an emergency department educational campaign for recognition of suicidal patients. The western journal of emergency medicine. 2012; 13(1): 41-50. 6. Shewan LG, Coats AJS. Ethics in the authorship and publishing of scientific articles. Int J Cardiol. 2010; 144(1): 1-2. PubMed

7. Verma SD, Davidoff DA, Kambhampati KK. Management of the agitated elderly patient in the nursing home: the role of the atypical antipsychotics. The Journal of clinical psychiatry. 1998; 19: 50-5.

8. Bogner JA, Corrigan JD, Bode RK, Heinemann AW. Rating scale analysis of the Agitated Behavior Scale. The Journal of head trauma rehabilitation. 2000; 15: 656-69.

9. Schmidtke A, Bille-Brahe U, DeLeo D, Kerkhof A, Bjerke T, Crepet P, et al. Attempted suicide in Europe: rates, trends and sociodemographic characteristics of suicide attempters during the period 1989-1992. Results of the WHO/EURO Multicentre Study on Parasuicide. Acta psychiatrica Scandinavica. 1996; 93(5): 327-38.

10. Serrano C, Silva MRE. A Review of Cardiopulmonary Research in Brazilian Medical Journals: Clinical, Surgical and Epidemiological Data. Clinics. 2010; 65(4): 441-5.

11. Rodham K, Hawton K, Evans E. Reasons for deliberate self-harm: comparison of self-poisoners and self-cutters in a community sample of adolescents. Journal of the American Academy of Child and Adolescent Psychiatry. 2004; 43(1): 80-7.

12. Briere J, Gil E. Self-mutilation in clinical and general population samples: prevalence, correlates, and functions. The American journal of orthopsychiatry. 1998; 68(4): 609-20. 13. Hickey L, Hawton K, Fagg J, Weitzel H. Deliberate self-harm patients who leave the accident and emergency department without a psychiatric assessment: a neglected population at risk of suicide. Journal of psychosomatic research. 2001; 50(2): 87-93.

14. Hendrix L, Verelst S, Desruelles D, Gillet JB. Deliberate self-poisoning: characteristics of patients and impact on the emergency department of a large university hospital. Emergency medicine journal. 2013: 30(1):e9

15. Othmer E, Othmer SC, Othmer JP. Psychiatric interview, History, and Mental Status Examination. In: BJ Sa- 
dock, VA Sadock; eds. Kaplan\&Sadock’s Comprehensive Textbook of Psychiatry. 8th edition. Philadelphia: LippincottWilliams \& Wilkins, 2005:849.

16. Wilson MP, Pepper D, Currier GW, Holloman GH Jr, Feifel D. The psychopharmacology of agitation: consensus statement of the american association for emergency psychiatry project Beta psychopharmacology workgroup. The western journal of emergency medicine. 2012; 13(1): 26-34. 17. Weiss S, Peterson K, Cheney P, Froman P, Ernst A, Campbell M. The Use of Chemical Restraints Reduces Agitation in Patients Transported by Emergency Medical Service. The Journal of emergency medicine. 2012: 43(5):820-8. 